\title{
Tendências da produção científica em odontologia no Brasil
}

\author{
Aldo Angelim Dias, ${ }^{1}$ Paulo Capel Narvai ${ }^{2}$ e Delane Maria Rêgo ${ }^{3}$
}

Como citar

Dias AA, Narvai PC, Rêgo DM. Tendências da produção científica em odontologia no Brasil. Rev Panam Salud Publica. 2008:24(1):54-60.

RESUMO Objetivo. Descrever a tendência da produção científica odontológica no Brasil, destacando-se a área de saúde bucal coletiva, nos primeiros anos do século XXI.

Métodos. Os resumos de trabalhos apresentados nas reuniões da Sociedade Brasileira de Pesquisa Odontológica (SBPqO) de 2001 a 2006 foram avaliados em termos do seu desenho metodológico (estudo agregado ou individuado; estudo observacional ou de intervenção; estudo transversal ou longitudinal), natureza geral (revisões bibliográficas, estudos com seres humanos ou pesquisas laboratoriais) e enquadramento em uma das 19 especialidades reconhecidas pelo Conselho Federal de Odontologia (CFO). Dos 10406 trabalhos apresentados nesse período, foram lidos 5203 (50,0\% do total).

Resultados. Quanto ao desenho metodológico, 87,5\% dos resumos eram do tipo operativo individuado e $12,5 \%$ do tipo agregado. Na classificação da natureza geral da pesquisa, $41,7 \%$ dos resumos tratavam de estudos com seres humanos. Os resumos restantes $(58,3 \%)$ tratavam de pesquisas laboratoriais in vitro $(31,1 \%)$, pesquisas laboratoriais in vivo $(23,6 \%)$ e revisões bibliográficas (3,6\%). Com relação às áreas de conhecimento do CFO, apenas cinco atingiram percentuais de ocorrência superiores a 10,0\%: dentística, periodontia, endodontia, odontopediatria e saúde coletiva.

Conclusões. A produção científica odontológica brasileira no período de 2001 a 2006 foi equilibrada, com crescente interesse na área de saúde bucal coletiva.

Palavras-chave Odontologia, pesquisa/tendências, Brasil.

A pesquisa odontológica no Brasil experimentou um aumento quantitativo significativo nos primeiros anos do sé-

1 Universidade Federal do Rio Grande do Norte (UFRN), Programa de Pós-Graduação, e Universidade de Fortaleza (UNIFOR). Correspondência: Rua Coronel Jucá 330, apto. 1202, Meireles, CEP 60170-320, Fortaleza, CE, Brasil. E-mail: aldo_ angelim@hotmail.com

2 Universidade de São Paulo (USP), Faculdade de Saúde Pública, São Paulo (SP), Brasil. E-mail: pcnarvai@usp.br

3 Universidade Federal do Rio Grande do Norte (UFRN), Programa de Pós-Graduação em Ciências da Saúde (PPGCSA) e Departamento de Odontologia, Natal (RN), Brasil. E-mail: regodm@ufrnet.br culo XXI, expresso pela maior divulgação em revistas especializadas e por apresentações em encontros científicos (1). No entanto, esse aumento não foi necessariamente acompanhado por uma melhora na qualidade do que se publica. Além disso, é comum que os resultados de uma determinada pesquisa, ainda que apresentados em encontros científicos, nunca cheguem aos periódicos, não se completando, dessa forma, o processo de produção-divulgaçãoapropriação de conhecimentos.
Em um ensaio sobre a qualidade dos artigos científicos publicados na área da saúde, Szklo (2) menciona vários fatores associados à má qualidade desse material, desde a interpretação errônea de dados e a análise equivocada de associações estatísticas até a ocorrência de problemas elementares, como resumos extensos demais e uso excessivo de abreviações. Nesse quesito, o adequado delineamento da pesquisa é também um fator decisivo e fundamental para o alcance dos objeti- 
vos, ainda que nenhum delineamento impeça erros (3).

A acessibilidade aos periódicos é outro fator a ser considerado. A informação consultada pelos cirurgiõesdentistas geralmente é definida pela facilidade de acesso, como apontado em estudo de Amorim et al.(4). As autoras concluíram que as três revistas mais consultadas são de fácil acesso, tratam de assuntos mais gerais e têm uma flexibilidade maior na escrita do texto.

O objetivo deste artigo é descrever as tendências das características quantitativas da produção científica odontológica no Brasil, destacando-se a área de saúde bucal coletiva, nos primeiros anos do século XXI. Para tanto, os resumos de trabalhos apresentados nas reuniões da Sociedade Brasileira de Pesquisa Odontológica ( $\mathrm{SBPqO}$ ) foram avaliados em termos de sua área de conhecimento e do tipo e delineamento da pesquisa.

\section{MATERIAIS E MÉTODOS}

O material investigado constituiu-se dos resumos dos trabalhos apresentados nas reuniões da $\mathrm{SBPqO}$ no período de 2001 a 2006. Dos 10406 trabalhos apresentados nesse período, foram lidos 5203 resumos, correspondendo a $50,0 \%$ do total. Foram lidos todos os resumos de numeração ímpar em cada seção em que se divide a grade de apresentação dos trabalhos durante o evento (5-10). Como o número de trabalhos apresentados a cada ano não foi o mesmo, exatamente a metade dos resumos de cada ano foi lida.

A SBPqO é um ramo da International Association for Dentistry Research (Associação Internacional para Pesquisa Odontológica, IADR) e se reúne anualmente desde 1983. As pesquisas apresentadas durante esses encontros anuais servem como referência para o perfil da pesquisa odontológica desenvolvida no país (11). Nesse evento, são apresentados trabalhos previamente selecionados, oriundos de todas as regiões do Brasil e abrangendo todas as áreas de conhecimento em odontologia. Admite-se, portanto, que é possível tomá-los como representativos do conjunto da produção científica odontológica no Brasil.

Os resumos foram lidos e classificados de acordo com dados coletados a partir de um formulário específico que incluía as seguintes categorias de classificação: desenho metodológico, natureza geral e pertinência a uma determinada área de conhecimento odontológico. Dentro dessas categorias, incluíam-se outras subcategorias, conforme descrito a seguir.

Para a definição do desenho metodológico, foi utilizada a classificação epidemiológica de Rouquayrol e Almeida Filho (12), que divide a pesquisa de acordo com: 1) o tipo operativo ou a natureza do grupo estudado; 2) a posição do investigador; e 3) a referência temporal. Essa proposta está de acordo com a classificação da pesquisa sugerida por Lilienfeld (13) e Miettinen (14) e vai ao encontro das classificações sugeridas por autores clássicos da área de epidemiologia, como Rothman e Greenland (15), e por autores da área de saúde bucal coletiva, como Antunes e Peres (16).

Para o tipo operativo foram utilizadas as categorias "agregado" e "individuado". A categoria agregado se refere a coletivos humanos necessariamente determinados por fatores sociais ou coletivos. São exemplos de agregados a população das escolas de um município ou o conjunto de habitantes de uma localidade. A categoria individuado se refere a estudos onde os sujeitos são analisados um a um, e não como grupo, podendo englobar pesquisas clínicas ou laboratoriais.

Quanto à posição do investigador, as pesquisas foram classificadas como "estudos observacionais" ou "estudos de intervenção", os quais determinam a participação passiva ou ativa, respectivamente, do pesquisador. Tal descrição pode ser utilizada em conjunto com as categorias empregadas para classificar o tipo operativo (agregado ou individuado). Ainda na classificação do desenho metodológico, os estudos foram agrupados quanto à referência temporal em "transversais" e "longitudinais".

Essas categorias, por permitirem inserção simultânea, possibilitam cruza- mentos, de modo que, por exemplo, os estudos de coorte ou prospectivos podem ser classificados como pesquisas individuadas, observacionais e longitudinais. A mesma classificação se aplica aos estudos de caso-controle (sendo estes retrospectivos). Já os ensaios clínicos podem ser classificados como estudos individuados, de intervenção e longitudinais.

Quanto à natureza geral, os estudos foram divididos em: 1) revisões bibliográficas (incluindo as revisões simples e as sistemáticas com ou sem metaanálise); 2) estudos com seres humanos (pesquisas clínicas ou com sujeito coletivo); e 3) pesquisas laboratoriais (in vivo, quando realizadas em animais, e in vitro).

A classificação das pesquisas segundo as áreas de conhecimento levou em consideração o seu enquadramento em uma das 19 especialidades reconhecidas pelo Conselho Federal de Odontologia (CFO) (17). Embora a especialidade "saúde bucal coletiva" extrapole, por sua natureza, o que se poderia denominar de campo odontológico, uma vez que se constitui também em uma área de conhecimento do campo mais geral da "saúde coletiva", neste estudo é considerada como uma especialidade odontológica para fins analíticos. Os resultados foram expressos como freqüência de cada categoria.

\section{RESULTADOS}

Quanto ao desenho metodológico, $87,5 \%$ dos resumos eram do tipo operativo individuado e $12,5 \%$ do agregado, o agregado. Com pequenas variações durante o período analisado, a freqüência dos estudos operativos individuados foi sempre acima de $80,0 \%$, com exceção do ano de 2005 (77,0\%). Em 2003, os estudos individuados alcançaram 97,0\% (tabela 1). No quesito "posição do investigador", 48,8\% foram estudos de observação. Nesses, por definição, o pesquisador não interveio diretamente no fluxo dos acontecimentos que tomou como objeto de pesquisa (tabela 2). Ainda quanto ao desenho metodológico, na dimensão "referência temporal" 52,9\% foram es- 
TABELA 1. Freqüência absoluta e relativa dos estudos ${ }^{a}$ apresentados nas reuniões da Sociedade Brasileira de Pesquisa Odontológica conforme tipo operativo, Brasil, 2001 a 2006

\begin{tabular}{lrrrrr}
\hline & \multicolumn{2}{c}{ Estudos agregados } & & \multicolumn{2}{c}{ Estudos individuados } \\
\cline { 2 - 3 } Ano & $n$ & $\%$ & & \\
\hline 2001 & 117 & 17,0 & 570 & $\% 3,0$ \\
2002 & 46 & 11,0 & 373 & 89,0 \\
2003 & 38 & 3,0 & 1214 & 97,0 \\
2004 & 98 & 11,0 & 797 & 89,0 \\
2005 & 170 & 23,0 & 572 & 87,0 \\
2006 & 181 & 15,0 & 1027 & 87,0 \\
Total & 650 & 12,5 & 4553 & \\
\hline
\end{tabular}

$\mathrm{a}_{n=5203}$

TABELA 2. Freqüência absoluta e relativa dos estudos ${ }^{a}$ apresentados nas reuniões da Sociedade Brasileira de Pesquisa Odontológica conforme posição do investigador, Brasil, 2001 a 2006

\begin{tabular}{lccccc}
\hline & \multicolumn{2}{c}{ Estudos observacionais } & & \multicolumn{2}{c}{ Estudos de intervenção } \\
\cline { 2 - 3 } \cline { 5 - 6 } Ano & $n$ & $\%$ & & & $\%$ \\
\hline 2001 & 298 & 43,5 & 389 & 56,5 \\
2002 & 187 & 44,6 & & 232 & 55,4 \\
2003 & 680 & 54,3 & 572 & 45,7 \\
2004 & 362 & 40,4 & 533 & 59,6 \\
2005 & 470 & 63,3 & 272 & 36,7 \\
2006 & 570 & 47,2 & 638 & 52,8 \\
Total & 2567 & 48,8 & & 2636 & 51,2 \\
\hline
\end{tabular}

a $n=5203$.

TABELA 3. Freqüência absoluta e relativa dos estudos ${ }^{a}$ apresentados nas reuniões da Sociedade Brasileira de Pesquisa Odontológica conforme referência temporal, Brasil, 2001 a 2006

\begin{tabular}{lccccc}
\hline & \multicolumn{2}{c}{ Estudos transversais } & & \multicolumn{2}{c}{ Estudos longitudinais } \\
\cline { 2 - 3 } Ano & $n$ & $\%$ & & \\
\hline 2001 & 395 & 57,5 & 292 & 42,5 \\
2002 & 174 & 41,5 & 245 & 58,5 \\
2003 & 576 & 46,0 & 676 & 54,0 \\
2004 & 353 & 39,5 & 542 & 60,5 \\
2005 & 378 & 51,0 & 364 & 49,0 \\
2006 & 574 & 47,5 & 634 & 52,5 \\
Total & 2450 & 47,1 & 2753 & 52,9 \\
\hline a $n=5$ 203. & & & &
\end{tabular}

tudos longitudinais e $47,1 \%$, transversais (tabela 3).

Com relação à segmentação das pesquisas nas áreas do conhecimento odontológico definidas pelo CFO (17), apenas cinco atingiram percentuais gia e traumatologia bucomaxilofaciais, ortodontia e estomatologia tiveram freqüências entre 4,7 e 9,8\%. As outras nove especialidades reunidas totalizaram 9,6\%. Nesse conjunto encontramse as cinco especialidades aprovadas pelo CFO em 2002: odontologia do trabalho, ortopedia funcional dos maxilares, disfunção temporomandibular e dor orofacial, odontologia para pacientes com necessidades especiais e odontogeriatria. A essas se somam radiologia odontológica e imaginologia, implantodontia, odontologia legal e prótese bucomaxilofacial (tabela 4).

$\mathrm{Na}$ classificação da natureza geral da pesquisa, $41,7 \%$ dos resumos tratavam de estudos com seres humanos. O peso maior nesse total, entretanto, foi dos estudos de natureza clínica quando comparados aos de base populacional. Os resumos restantes $(58,3 \%)$ tratavam de pesquisas laboratoriais in vitro $(31,1 \%)$, pesquisas laboratoriais in vivo $(23,6 \%)$ e revisões bibliográficas $(3,6 \%)$ (tabela 5).

\section{DISCUSSÃO}

Desde meados dos anos 1970, um representativo campo intelectual se formou no Brasil, no qual as ciências sociais e a área de ensino e educação tomaram como tema a saúde, que, por sua vez, recebeu a influência de conjunturas sociais, políticas e institucionais. A esse respeito, Nunes (18) pondera que há um campo aberto para estudos sobre a construção científica na área das ciências sociais em saúde. Isso vem sendo realizado por meio de estudos que buscam fundamentação na sociologia, história e filosofia da ciência, permitindo uma análise mais aprofundada da produção científica. Apesar de esse crescimento contemplar praticamente todas as áreas de conhecimento do campo mais geral da saúde, nota-se que, na odontologia, ele tem acontecido de forma mais lenta e gradual.

Ainda são poucos os relatos sobre a quantidade e a qualidade dos trabalhos científicos realizados na área odontológica no Brasil, embora haja um crescimento gradativo do interesse por esses estudos nos últimos anos, permitindo 
TABELA 4. Freqüências absoluta e relativa dos estudos ${ }^{\mathrm{a}}$ apresentados nas reuniões da Sociedade Brasileira de Pesquisa Odontológica conforme área de conhecimento, Brasil, 2001 a 2006

\begin{tabular}{llr}
\hline \multicolumn{1}{c}{ Área } & $n$ & $\%$ \\
\hline Dentística & 665 & 12,8 \\
Periodontia & 609 & 11,7 \\
Endodontia & 593 & 11,4 \\
Odontopediatria & 562 & 10,8 \\
Saúde coletiva & 547 & 10,5 \\
Patologia & 510 & 9,8 \\
Prótese buco-dentária & 385 & 7,4 \\
Cirurgia & 317 & 6,1 \\
Ortodontia & 271 & 5,2 \\
Estomatologia & 245 & 4,7 \\
Outras áreas & 499 & 9,6 \\
$\quad$ Total & 5203 & 100,0 \\
\hline a & &
\end{tabular}

TABELA 5. Freqüência absoluta e relativa dos estudos ${ }^{\mathrm{a}}$ apresentados nas reuniões da Sociedade Brasileira de Pesquisa Odontológica conforme a natureza geral da pesquisa, Brasil, 2001 a 2006

\begin{tabular}{lrr}
\hline \multicolumn{1}{c}{ Natureza da pesquisa } & $n$ & $\%$ \\
\hline Estudos em humanos & 2170 & 41,7 \\
$\quad$ Pesquisas clínicas & 1572 & 30,2 \\
Pesquisas com sujeitos coletivos & 598 & 11,5 \\
Pesquisas laboratoriais & 2846 & 54,7 \\
$\quad$ In vitro & 1618 & 31,1 \\
In vivo & 1228 & 23,6 \\
Revisões bibliográficas & 187 & 3,6 \\
$\quad$ Total & 5203 & 100,0 \\
\hline${ }^{a} n=5$ 203. & &
\end{tabular}

traçar um diagnóstico mais preciso do que se produz em nosso país. Para esta pesquisa, optamos por utilizar o mesmo campo de ação empregado por Cormack e Silva-Filho (11). Na análise da produção apresentada na Reunião Anual da SBPqO de 1997, os autores concluíram que a pesquisa odontológica no Brasil estava voltada para assuntos que tratavam dos materiais de uso odontológico, aspectos biológicos e técnicas clínico-cirúrgicas. Tais temáticas corresponderam a 88,3\% dos trabalhos daquele ano. Apenas 11,7\% das pesquisas versaram sobre assuntos de cunho social. A participação do setor privado no financiamento dessa produção foi considerada inexpressiva
$(1,7 \%)$; as instituições públicas, por sua vez, despendiam suas verbas de forma desvinculada da área social. Os autores enfatizaram o fato de que, apesar de o financiamento ser essencialmente realizado por instituições públicas, a grande maioria das pesquisas se orientava ao estudo de materiais odontológicos com fins terapêuticos com pouco ou quase nenhum alcance para a maior parte da população (11). Já no presente estudo, observamos um predomínio de pesquisas clínicas ou com sujeitos coletivos $(41,7 \%)$. Entretanto, dentro dessa categoria, a maior contribuição foi das pesquisas de natureza clínica $(30,2 \%)$ em relação às pesquisas com sujeitos coletivos $(11,5 \%)$.
O alto percentual de pesquisas laboratoriais $(54,7 \%)$ deve ser visto com atenção, já que muitos desses estudos englobam benefícios que podem ser aplicados diretamente à coletividade. É o caso, por exemplo, das pesquisas que visam a reduzir os custos na área da prevenção, com o desenvolvimento de novos produtos para escovação ou de materiais restauradores mais acessíveis. Embora esses estudos sejam realizados em um ambiente laboratorial, podem ter aplicação na área de saúde coletiva $(19,20)$. Antunes e Peres (16) também afirmam que a observação clínica e a pesquisa laboratorial se integram aos estudos epidemiológicos, formando um tripé que sustenta os conhecimentos utilizados para os programas de saúde. Para Gadelha (21), o desenvolvimento da sociedade está intrinsecamente ligado ao conhecimento, à pesquisa e à expansão do sistema de saúde em sua integralidade, incluindo estudos que possam trazer benefícios diretos ou indiretos para a saúde bucal coletiva.

Quanto ao desenho metodológico, na dimensão referência temporal, chama a atenção o fato de que 52,9\% dos estudos tenham sido classificados como longitudinais. Esse índice se justifica pelo alto número de estudos laboratoriais $(54,7 \%)$ e pesquisas clínicas com seres humanos $(30,2 \%)$ (tabela 5), que, geralmente, envolvem seguimento ao longo de um determinado espaço de tempo.

Os resultados mostram que as áreas que tratam diretamente das doenças bucais de maior prevalência (cárie e doença periodontal) também foram o foco mais freqüente da pesquisa. É válido assinalar, porém, que houve uma proximidade dos valores de freqüência relativa (entre 10,5 e 12,8\%) obtidos pelas cinco primeiras áreas, que ainda incluíam a endodontia, a odontopediatria e a saúde coletiva. Destaca-se a posição ocupada pela saúde coletiva $(10,5 \%)$, superando todas as outras especialidades subseqüentes. Sua posição limítrofe em relação à patologia $(9,8 \%)$, especialidade que estuda aspectos histopatológicos das alterações do complexo bucomaxilofacial mostra, de certa forma, uma tendência de re- 
versão da preocupação dos grupos científicos em pesquisar apenas o dano ou a lesão para estudar outros aspectos que envolvem o adoecer humano, a sua prevenção e a promoção da saúde.

Utilizando como categorias as áreas de conhecimento do Conselho Nacional de Desenvolvimento Científico e Tecnológico (CNPq) do Ministério da Ciência e Tecnologia do Brasil, ao invés das especialidades do $\mathrm{CFO}$, como no presente estudo, Cavalcanti et al. (22) obtiveram resultados semelhantes, com a odontologia preventiva e social (equivalente neste estudo à saúde coletiva) em segundo lugar entre as áreas mais estudadas $(8,5 \%)$, tendo como grupo de análise os trabalhos apresentados na $20^{\mathrm{a}}$ Reunião Anual da SBPqO. No estudo citado, as outras áreas onde mais houve pesquisa foram materiais dentários, endodontia, odontopediatria e radiologia, nesta ordem.

No presente estudo, o mesmo raciocínio usado para explicar a posição ocupada pela saúde coletiva pode também ser utilizado para interpretar o comportamento das três áreas seguintes: prótese $(7,4 \%)$, cirurgia $(6,1 \%)$ e ortodontia (5,2\%). Essas áreas sempre despertaram o interesse dos cirurgiões-dentistas, mostrando-se, nesta avaliação, ainda com ocorrência importante nos grupos estudados. Se, durante décadas, a reabilitação, a cirurgia e a ortodontia interceptora ou corretiva prevaleceram na profissão odontológica, tanto como objeto de suas práticas quanto como objeto de investigação científica, os resultados desta pesquisa indicam uma nova tendência.

A estomatologia, uma área ainda pouco explorada da odontologia (apenas 369 cirurgiões-dentistas, ou 0,17\% do total de profissionais, são registrados nessa especialidade no CFO) (23), tem apresentado um aumento significativo no número de pesquisas nos últimos anos, o que justifica estar entre as 10 áreas mais estudadas.

Todas as cinco especialidades registradas no CFO em 2002 ficaram entre as áreas menos estudadas. A baixa ocorrência provavelmente se deve a dois fatores: a ausência de disciplinas correlatas nos cursos de graduação em odontologia e os poucos cursos de pós-graduação lato e stricto sensu com foco nessas áreas.

Amorim (24) obteve resultados semelhantes aos do presente estudo ao analisar os três periódicos brasileiros mais consultados pelos cirurgiõesdentistas, entre 1990 e 2004. A autora encontrou que $52,73 \%$ dos artigos nesses periódicos estão concentrados em apenas cinco áreas: dentística $(13,41 \%)$, saúde coletiva $(11,25 \%)$, endodontia $(11,24 \%)$, estomatologia/ patologia $(8,95 \%)$ e prótese $(7,89 \%)$. Destaca-se novamente a participação significativa da área de saúde coletiva, a segunda mais freqüente em periódicos que, por sua vez, concentravam $52,74 \%$ do acesso e utilização pelos cirurgiões-dentistas brasileiros.

Os resultados aqui descritos vão ao encontro da observação de Barros (25) e Birman (26), que afirmam que o progresso observado na saúde coletiva reflete a demanda crescente das universidades e principalmente do Sistema Único de Saúde. Embora incipiente, tal evolução quantitativa acena para uma nova realidade, na qual o interesse pelo social vem se consolidando como objeto de estudo nas instituições de ensino e pesquisa.

Narvai e Almeida (27) avaliaram a produção científica brasileira na área de odontologia social e preventiva no período de 1986 a 1993 e a associação dessa produção com o sistema de saúde e as políticas de saúde. Foram examinados 386 artigos, publicados em 19 periódicos. Embora o trabalho tenha se restringido à odontologia social e preventiva, é relevante destacar que os autores concluíram que cerca de $75 \%$ da produção nessa área tiveram origem em universidades públicas.

Em comparação com a pesquisa de Narvai e Almeida, na qual 56,7\% dos artigos eram originais e outros 30,3\% traziam revisões de literatura e ensaios, neste estudo as revisões ficaram em 3,6\%, já que algumas categorias das reuniões da $\mathrm{SBPqO}$ foram mais dirigidas aos estudos sobre educação em saúde, com maior tendência de se apresentarem naquela classificação.

Embora seja importante, em função de sua rapidez e chegada imediata ao público-alvo, a apresentação de trabalhos em congressos e eventos técnico-científicos, segundo Población e Duarte (28), ainda se reveste de um tratamento menos formal quando comparada à divulgação mais criteriosa, representada pela publicação em revistas científicas (29). Assim, cabe enfatizar que a divulgação em periódicos possibilita que toda uma comunidade, que lida com a construção e o progresso do conhecimento, possa se beneficiar dos resultados de cada investigação científica, na medida em que faz alavancar esse progresso e o submete à apreciação de pares acadêmicos.

Foratini (30) assinala que, no Brasil, muitos periódicos não preenchem critérios de qualidade como impacto, competitividade e internacionalidade. Para Cury (31), a odontologia brasileira ainda está em estado inicial, não tendo incorporado os critérios internacionais. No entanto, o autor admite que a busca por parâmetros claros e objetivos de qualidade deve ser a tônica dos periódicos brasileiros.

Para Narvai (32), o aumento do número de publicações em saúde bucal coletiva, que se expressa também na edição de livros, ainda não é suficiente para consolidar essa área como uma das mais produtivas, embora essa pareça ser uma tendência a curto e médio prazo.

Nessa perspectiva, Mashelkar (33) afirma que a força econômica de um país é confrontada com sua capacidade autóctone de pesquisa. Dados do CNPq (34) indicam que o setor saúde, no período de 2000 a 2004, foi responsável por $27 \%$ das linhas e 33,2\% dos grupos de pesquisa. Com relação ao número de pesquisadores, a participação da área de saúde chegou a 32,9\% do total de pesquisadores da base do CNPq no ano de 2004. Esses números refletem uma participação importante em todas as linhas de pesquisa, incluindo a saúde pública, responsável por $13,2 \%$ dos estudos realizados naquele ano. $\mathrm{O}$ aumento da produção de pesquisa gera mais necessidade de divulgação no meio científico.

Merece registro o fato de o CNPq ter firmado, em 2004, uma parceria com o Ministério da Saúde, por meio da qual 
1 milhão de reais foram destinados especificamente ao financiamento de projetos de pesquisa no campo da saúde bucal coletiva (35). O edital $\mathrm{n}^{\mathrm{o}}$ 038/2004 do CNPq/Ministério da Saúde/Departamento de Ciência e Tecnologia (CNPq/MS-DECIT) contemplou 10 linhas de apoio sobre o tema, desde modelos de atenção e serviços de saúde bucal até impactos epidemiológicos e estudos de risco ocupacional em profissionais da área. A iniciativa interministerial emergiu num momento em que a saúde bucal coletiva ganha destaque na discussão das políticas públicas de saúde.

Silveira (36) pondera que os desafios atuais da pesquisa odontológica brasileira remetem ao incremento da publicação em periódicos nacionais e estrangeiros e à "aplicação racional" dos resultados "visando à melhoria de qualidade da saúde das populações".

\section{CONCLUSÕES}

Conclui-se que, no período de 2001 a 2006, a pesquisa odontológica no Brasil se caracterizou pelas seguintes tendências:

- predomínio, quanto ao desenho metodológico, dos estudos de natureza individuada, típicos de pesquisas com enfoque clínico, para o estudo de materiais e com impacto limitado, mas não excludente, para as coletividades humanas;

- apesar da pouca diferença percentual, predomínio dos estudos de natureza intervencionista sobre os observacionais, e dos estudos longitudinais sobre os transversais;

- freqüência maior, quanto à área do conhecimento, de pesquisas nas áreas da dentística, da periodontia, da endodontia e da odontopediatria e participação significativa da área de conhecimento da saúde bucal coletiva, que correspondeu à quinta área com maior produção dentre 19 especialidades;

- quanto à natureza geral, freqüência maior de estudos laboratoriais com relação àqueles em humanos. As pesquisas que usaram sujeitos coletivos como unidade de análise foram bem menos freqüentes do que as pesquisas eminentemente clínicas.

A análise das reuniões da $\mathrm{SBPqO}$ mostrou que, não obstante as reconhecidas dificuldades encontradas para produzir conhecimento na área da saúde em países em vias de desenvolvimento, houve uma equilibrada produção no campo odontológico brasileiro no período de 2001 a 2006, com crescente interesse na área de saúde bucal coletiva.

\section{REFERÊNCIAS}

1. Souza PR. O crescimento da produção científica. Folha de São Paulo 2002. Novembro 06:3.

2. Szklo M. Quality of scientific articles. Rev Saude Publica. 2006;40(N Esp):30-6.

3. Ambrosano GM, Reis AF, Giannini M, Pereira AC. Use of statistical procedures in Brazilian and international dental journals. Braz Dent J. 2004;15(3):231-7.

4. Amorim KPC, Alves MSCF, Germano RM. A construção do conhecimento na odontologia: a produção científica em debate. Acta Cir Bras. 2005;20(supl 1):12-5.

5. Sociedade Brasileira de Pesquisa Odontológica (SBPqO). Resumos dos trabalhos. Em: $18^{\mathrm{a}}$ Reunião Anual da Sociedade Brasileira de Pesquisa Odontológica. São Paulo: SBPqO; 2001.

6. Sociedade Brasileira de Pesquisa Odontológica (SBPqO). Resumos dos trabalhos. Em: $19^{\text {a }}$ Reunião Anual da Sociedade Brasileira de Pesquisa Odontológica. São Paulo: SBPqO; 2002.

7. Sociedade Brasileira de Pesquisa Odontológica (SBPqO). Resumos dos trabalhos. Em: 20a Reunião Anual da Sociedade Brasileira de Pesquisa Odontológica. São Paulo: SBPqO; 2003.

8. Sociedade Brasileira de Pesquisa Odontológica (SBPqO). Resumos dos trabalhos. Em: 21 ${ }^{\text {a }}$ Reunião Anual da Sociedade Brasileira de Pesquisa Odontológica. São Paulo: SBPqO; 2004.

9. Sociedade Brasileira de Pesquisa Odontológica (SBPqO). Resumos dos trabalhos. Em: 22a
Reunião Anual da Sociedade Brasileira de Pesquisa Odontológica. São Paulo: SBPqO; 2005.

10. Sociedade Brasileira de Pesquisa Odontológica (SBPqO). Resumos dos trabalhos. Em: $23^{\mathrm{a}}$ Reunião Anual da Sociedade Brasileira de Pesquisa Odontológica. São Paulo: SBPqO; 2006.

11. Cormack EF, Silva-Fo. CF. A pesquisa científica odontológica no Brasil. Rev Assoc Paul Cir Dent. 2000;54(3);242-7.

12. Rouquayrol MZ, Almeida-Fo NA. Elementos de metodologia epidemiológica. Em: Rouquayrol MZ, Almeida-Fo NA. A epidemiologia e a saúde. $6^{\mathrm{a}}$ ed. Rio de Janeiro: MEDSI; 2003. Pp. 149-77.

13. Lilienfeld A. Foundations of epidemiology. Nova Iorque: Oxford; 1976.

14. Miettinen O. Theoretical epidemiology. Nova Iorque: Wiley and Sons; 1976.

15. Rothman K, Greenland S. Modern epidemiology. $2^{\mathrm{a}}$ ed. Filadélfia: Lippincott and Raven; 1988.

16. Antunes JLF, Peres MA. Epidemiologia da saúde bucal. Rio de Janeiro: Guanabara Koogan; 2006

17. Conselho Federal de Odontologia (CFO). Consolidação das normas para procedimentos nos Conselhos de Odontologia. Rio de Janeiro: Conselho Federal de Odontologia; 2005.

18. Nunes ED. A trajetória das ciências sociais em saúde na América Latina: revisão da produção científica. Rev Saude Publica. 2006; 40 (N Esp):64-72.
19. Wolff $D$, Joerss D, Dörfer CE. In vitro-cleaning efficacy of interdental brushes with different stiffness and different diameter. Oral Health Prev Dent. 2006;4(4):279-85.

20. Ferreira FM, Vale MPP, Jansen WC, Paiva SM, Pordeus IA. Perfomance of Brazilian and imported glass ionomer cements used in Atraumatic Restorative Treatment (ART) regarding microleakage in primary molars. J Appl Oral Sci. 2006;14(5):312-8.

21. Gadelha CAG. Desenvolvimento, complexo industrial da saúde e política industrial. Rev Saude Publica. 2006;40(N Esp):11-23.

22. Cavalcanti AL, Melo TRNB, Barroso KMA, Souza FEC, Maia AMA, Silva ALO. Perfil da pesquisa científica em Odontologia realizada no Brasil. Pesqui Bras Odontopediatria Clin Integr. 2004;4(2):99-104.

23. Conselho Federal de Odontologia (CFO). Dados do CFO: profissionais por especialidade. Disponível em: www.cfo.org.br. Acessado em dezembro de 2006.

24. Amorim KPC. A (bio)ética e a odontologia: os (des)caminhos de uma formação humana [tese]. Natal: Universidade Federal do Rio Grande do Norte; 2006.

25. Barros AJD. Produção científica em saúde coletiva: perfil dos periódicos e avaliação pela Capes. Rev Saude Publica. 2006;40(N Esp): 43-9.

26. Birman EG. Rumos da pesquisa odontológica [editorial]. Pesqui Odontol Bras. 2002;16(4):1.

27. Narvai PC, Almeida ES. O sistema de saúde e as políticas de saúde na produção científica 
odontológica brasileira no período 1986-1993. Cad Saude Publica. 1998;14(3):513-21.

28. Población DA, Duarte JG. Comunicação da informação científica entre pesquisadores. Intercom: Rev Bras Com. 1989;12(61):47-61.

29. Volpato GL, Freitas EG. Desafios na publicação científica. Pesqui Odontol Bras. 2003; 17(1):49-56.

30. Forattini OP. A internacionalidade da ciência [editorial]. Rev Saude Publica. 1997;31(2):115.

31. Cury JA. The evolution of dental research in Brazil. Braz Oral Res. 2004;18(2):1.

32. Narvai PC. Saúde bucal coletiva: caminhos da odontologia sanitária à bucalidade. Rev Saude Publica. 2006;40(N Esp):141-7.
33. Mashelkar RA. Nation building through science and technology: a developing world perspective. 10th Zuckerman Lecture. Innovation Strategy Today. 2005;1(1):16-32.

34. Brasil, Conselho Nacional de Desenvolvimento Científico e Tecnológico (CNPq). Diretório dos grupos de pesquisa no Brasil. Censos 2004-2002-2000 e base corrente. Disponível em http://dgp.cnpq.br/censo 2004. Acessado em dezembro de 2006.

35. Brasil, Conselho Nacional de Desenvolvimento Científico e Tecnológico (CNPq). Edital MCT/SCTIE/DECIT/MS/CNPq n ${ }^{\circ} 038 / 2004$. Seleção pública de propostas sobre saúde bucal. Disponível em: http://memoria.cnpq. br/servicos/editais/ct/edital_0382004_cnpq. htm. Acessado em dezembro de 2006.

36. Silveira FRX. Pesquisa odontológica e qualidade de vida. Pesqui Odontol Bras. 2003; 17(2):3.

Manuscrito recebido em 13 de outubro de 2007. Aceito em versão revisada em 15 de fevereiro de 2008.

ABSTRACT Objective. To describe dental research trends in Brazil (especially population-based oral health) in the early Twenty-first Century.

\section{Scientific output trends in oral health in Brazil}

Methods. The abstracts of studies presented at meetings of the Brazilian Society for Dental Medicine Research (Sociedade Brasileira de Pesquisa Odontológica) from 20012006 were assessed in terms of methodological design (aggregate or population-based and individual-based studies, observational and intervention studies, cross-sectional and longitudinal studies); general type (literature review, studies with human subjects, and laboratory studies); and classification into one of the 19 specialty categories recognized by the Brazilian Federal Dentistry Council. Of the 10406 abstracts presented in this period, 5203 (50\%) were reviewed.

Results. Concerning methodological design, $87.5 \%$ of the abstracts referred to individual-based studies, whereas $12.5 \%$ were of aggregate studies. Concerning the general category, $41.7 \%$ referred to studies with human subjects. The remaining abstracts $(58.3 \%)$ described in vitro $(31.1 \%)$ or in vivo $(23.6 \%)$ laboratory research and literature reviews $(3.6 \%)$. Concerning the Council's specialty categories, only five had a frequency higher than $10.0 \%$ : esthetic dentistry, periodontics, endodontics, pediatric dentistry, and population-based oral health.

Conclusions. Brazil's scientific output in the field of oral health for the period 20012006 was balanced, with increasing interest in the area of population-based oral health.

Key words Dentistry, research/trends, Brazil. 